\title{
25 Research Square

\section{Conditioned Excitation And Inhibition of Short Latency Gamma Band Oscillations In Early Visual Cortex During Fear Learning In Humans}

\section{Alejandro Santos-Mayo}

Universidad Complutense de Madrid

Javier de Echegaray

Universidad Complutense de Madrid

Stephan Moratti ( $\nabla$ smoratti@ucm.es )

Universidad Complutense de Madrid

\section{Research Article}

Keywords: oscillations, sinus grating, neurobiological, latency

Posted Date: June 29th, 2021

DOI: https://doi.org/10.21203/rs.3.rs-646537/v1

License: (c) (i) This work is licensed under a Creative Commons Attribution 4.0 International License.

Read Full License 


\section{Abstract}

Over the course of evolution the human brain has been shaped to prioritize cues that signal potential danger. Thereby, the brain does not only favor specie-specific prepared stimulus sets such as snakes or spiders, but can learn associations between new cues and aversive outcomes. One important mechanism to achieve this is associated with learning induced plasticity changes in sensory cortex that optimizes the representation of motivationally relevant sensory stimuli. Animal studies have shown that the modulation of gamma band oscillations predicts cholinergic driven plasticity changes in sensory cortices shifting neurons' responses to fear relevant features as acquired by Pavlovian fear conditioning. Here, we report conditioned excitatory and inhibitory gamma band modulations in humans during fear conditioning of orthogonally oriented sinus gratings representing fear relevant and irrelevant conditioned cues, respectively. Thereby, pairing of a sinus grating with an aversive loud noise not only increased short latency (during the first $180 \mathrm{~ms}$ ) evoked visual gamma band responses, but was also accompanied by strong gamma power reductions for the fear irrelevant control grating. The current findings will be discussed in the light of recent neurobiological models of cholinergic driven plasticity changes in sensory cortices and classic learning models such as the Rescorla-Wagner framework.

\section{Introduction}

Discriminating potentially harmful stimuli from background noise, such as detecting a snake hidden in the grass, is crucial for survival. During the course of evolution specie-specific stimulus categories have been evolved, that are innately perceptually prioritized. For example, in humans stimulus categories such as insects (e. g. spiders), reptiles (e. g. snakes) and highly social relevant stimuli (e.g. fearful faces) represent such to-be-prepared cues ${ }^{1-3}$. However, in ever-changing environments organisms also must be capable to learn to perceptually prioritize new stimuli that represent or alert danger and do not form part of the evolutionary prepared stimulus sets. For example, previously innocuous stimuli that have been paired with aversive events (e. g. a loud noise burst or a mild electric shock) can acquire the same motivational relevance as natural motivators; a process called Pavlovian fear conditioning. Importantly, stimuli that acquired fear relevance by learning are also perceptually favored as these stimuli also evoke autonomous reactions associated with orienting responses and modulate neural gain in early sensory cortices $^{4-6}$.

Inspired by animal models in the auditory domain, recently there has been a great interest in how shortterm plasticity changes in the human visual cortex alter the sensory representation of visual conditioned stimuli during associative learning, a process probably mediated by cholinergic modulation of disinhibitory mechanisms ${ }^{7-9}$. From a behaviorally adaptive point of view, increasing the neural gain in sensory systems guarantees better detection of acquired fear relevant stimuli among other potential distractors. However, best discrimination between fear relevant and irrelevant stimuli would be achieved not only by facilitating the neural processing of fear associated stimuli, but also by a suppression of neural activity of fear irrelevant stimuli. For example, in humans it has been shown that orientation sensitive visual cortex representations of Gabor patches that are similarly orientated as a fear relevant 
Gabor patch are suppressed possibly by retuning of orientation selectivity of visual cortex neurons ${ }^{10}$. Further, a recent MEG study has shown that during the course of fear conditioning neural gain increases for a fear relevant visual stimulus was accompanied by the inhibition of neural activity for the fear irrelevant cue ${ }^{5}$. These studied used steady state visual evoked potentials (ssVEPs, EEG) or fields (ssVEFs, $M E G$ ) that represent evoked neural oscillations driven by a visual flicker at a certain frequency. Steady state responses have the advantage of high signal to noise ratios but lack sufficient time resolution in order to determine if these excitatory and inhibitory neural gain modulations occur early in lower tiers of the visual cortex. Additionally, these studies showed rather late latency effects on steady state power changes evoked by flickering stimuli just before US onset ${ }^{6}$. Further, ssVEPs or ssVEFs represent reentrant oscillatory activity from higher to lower order visual cortex and it is difficult to detangle at which processing stage the aforementioned neural gain modulations occur ${ }^{11}$.

Here, we extent these previous findings by investigating if such opposing adaptive neural gain changes for fear relevant and irrelevant visual stimuli, that guarantee best stimulus discrimination, occur during very early processing stages in the visual cortex. Thereby, early neuromagnetic evoked gamma band oscillations usually observed during visual processing ${ }^{12,13}$ were recorded with MEG during a delayed discriminative Pavlovian fear conditioning procedure. Thereby, two orthogonally oriented visual sinus gratings served as the conditioned (CS+) and the control stimulus (CS-). After a habituation phase in that no CS had been paired with an aversive loud acoustic noise (unconditioned stimulus; US), only the CS + was paired with the US during CS-US association acquisition. Finally, during an extinction phase, none of the conditioned stimuli were paired with the US (see Fig. 1A and Methods for a detailed description).

Neuromagnetic evoked gamma band responses to such sinus patches represent early stages of visual information processing in humans ${ }^{14,15}$. Further, it has been shown that increased gamma band oscillations in sensory cortices predict sharpened representation of the CS + by plasticity changes such as shifts in frequency tuning of auditory cortex neurons during fear conditioning ${ }^{16-18}$. Similarly, gamma power increases support cholinergic driven changes in orientation selectivity of visual cortex neurons $7,19,20$. Therefore, neuromagnetic gamma band modulations as recorded by MEG in humans represent a good proxy for these plasticity changes. Shifts of orientation tuning in visual cortex towards to the CS + orientation and away from the CS- patch orientation could be reflected by gamma power increases and decreases, respectively. As efficient discrimination between fear relevant (CS+) and irrelevant information (CS-) should occur fast, we hypothesize that such excitatory and inhibitory gamma power related plasticity changes will be reflected by early evoked gamma band activity modulations.

Further, cortical source estimation of the corresponding neuromagnetic signal changes should localize to the lower tier of the visual cortex. Critically, reduced gamma band responses for the fear irrelevant stimulus should increase to baseline levels during fear extinction learning in order not to be attributable to habituation processes as a result of repeated stimulus presentation. Taken together, short latency opposing gamma power modulations at low levels of the visual system would represent short term 
plasticity processes that guarantee fast adaptive visual discrimination of acquired motivational information in humans.

\section{Results}

Unawareness of conditioning and levels of vigilance.

In order to maintain a certain level of the participants' attention and to prevent developing awareness of the CS-US contingency (the aware detection of the rule that the CS + predicts the US), volunteers had to respond to random color changes of a centered dot (black to grey) during the inter-stimulus interval. The total detection rate confirmed a high state of vigilance during the experiment (total: $95.56 \% \pm 8.32 \mathrm{~s}$. e. m.). The CS-US contingency was not detected by any of the participants as by the end of the experiment no one $(\mathrm{N}=30)$ could indicate which of the two Gabor patches (CS + or CS-) preceded the administration of the US.

\section{Evoked neuromagnetic gamma band responses.}

We hypothesized that best discriminative representations of the fear relevant CS + and fear irrelevant CSin visual cortex will be associated with early evoked oscillatory gamma band increases for the CS + and decreases for the CS- during the learning phase of the experiment compared to baseline conditions (habituation and extinction). Therefore, a time-frequency analysis was conducted at occipital and parietal sensors, usually showing an early gamma band response during visual processing (see Methods section). Further, a facilitation of visual processing during acquisition of the CS-US association for the CS + and an inhibition of CS- related activity should be best represented by an increase of gamma band power differences between the CS + and CS- from habituation to acquisition phases followed by a decrease of power differences for extinction trials. Therefore, a quadratic contrast (see Methods for details) across experimental phases was fitted to the CS + and CS- power differences, thus testing the interaction between quadratic CS + and CS- gamma power changes and experimental phase. The significance of this contrast was evaluated using a non-parametric permutation procedure (see Methods).

A sensor cluster at medial occipital sensors indicated a statistically significant quadratic contrast across experimental phases for the CS + and CS- differences (cluster-based permutations, summed $F=6536, p<$ 0.05 ; best fit at gradiometers MEG $2142+2143$, frequency $52.2 \mathrm{~Hz}$, time $0.1 \mathrm{~s}, \mathrm{~F}(1,29)=17.8, p<0.001$, $\eta^{2}=0.381$ ). The topography of the resulting cluster is shown in Fig. 1B. Figure $1 \mathrm{C}$ depicts the five timefrequency power differences between the CS + and CS- across significant sensors for each experimental phase (habituation block II, acquisition block I and II, and extinction block I and II). The quadratic effect spanned from stimulus onset to $180 \mathrm{~ms}$ and ranged between $18.9 \mathrm{~Hz}$ to $71 \mathrm{~Hz}$. The CS+ / CS- difference was biggest during the second block of acquisition (see Fig. 1D) and returned to habituation levels during extinction. The paired observation plots for each individual subject in Fig. 1D indicated a robust quadratic pattern of CS + and CS- gamma power differences across experimental phases. 
Critically, the quadratic modulation of CS + and CS- differences across experimental phases was not only driven by a progressive increase of gamma band responses during acquisition for the CS+ (see red line in Fig. 1E), but also by CS- gamma band response inhibition (see blue line in Fig. 1E). Accordingly, CS + mean gamma power across significant sensor by frequency by time clusters (see above) followed a quadratic response profile across experimental conditions (quadratic fit: $F(1,29)=13.89, p<0.001, \eta^{2}=$ $0.324)$. In contrast, the CS- condition was characterized by a progressive decrease of gamma responses during acquisition and a return to baseline during extinction (quadratic fit: $F(1,29)=44.46, p<0.001, \eta^{2}=$ 0.605).

Cortical source localization of gamma response interaction.

Although the hypothesized effects were observed at posterior MEG sensors, a linearly constrained minimum variance (LCMV) beamformer was applied (using all gradiometers) in order to localize the quadratic gamma power difference (CS + vs. CS-) modulation in cortical source space. Time resolved LCMV beamformer source waveforms between stimulus onset and $180 \mathrm{~ms}$ after stimulus presentation were submitted to an FFT and mean power between $18.9 \mathrm{~Hz}$ and $71 \mathrm{~Hz}$ was extracted at cortical source locations (baseline corrected to a corresponding time window in the pre-stimulus interval, see Methods). The same quadratic contrast and permutation procedure as for the sensor level analysis was fitted to the CS + and CS- power differences across experimental conditions. A significant source cluster revealed enhanced gamma power differences (CS + minus CS-) during acquisition from primary visual cortex to lateral secondary visual areas in the right hemisphere (cluster-based permutation, summed $F=747 ; p<$ 0.05 , maximum $F$ value: $F(1,29)=21.891, p<0.001, \eta^{2}=0.43$; Figs. $\left.2 A \& 2 B\right)$. Therefore, the LCMV beamformer confirmed that the observed sensor level effects were attributable to the lower tiers of the visual cortex.

\section{Discussion}

Here, we show that conditioned excitatory and inhibitory neural gain modulations in the human visual cortex were greatest around $100 \mathrm{~ms}$ after stimulus onset for acquired fear relevant and irrelevant stimuli, respectively. This was reflected by corresponding evoked gamma band power modulations between 18.9 $\mathrm{Hz}$ and $71 \mathrm{~Hz}$ in lower tiers of the visual cortex. Thereby, oscillatory neuromagnetic gamma band activity not only increased for the CS + during the acquisition phase of the CS-US association, but also decreased for the CS-. Critically, both, CS + and CS- related evoked gamma powers returned to pre-conditioning levels during extinction. Our findings not only replicate previous observations of excitatory and inhibitory shortterm plasticity changes in the visual cortex by learning, but significantly extent these reports by demonstrating that these changes occur very fast within the first $180 \mathrm{~ms}$ peaking at around $100 \mathrm{~ms}$ after stimulus onset at early stages of the visual cortex. Our findings are in line with the observation that evoked short latency gamma power changes in the visual cortex represent very early visual processing stages $^{14,15}$. In contrast, we did not find any gamma power modulations for induced gamma band responses (see supplementary information). 
Early stages of visual processing could not be directly assessed by previous studies that showed opposing neural gain modulations in the visual cortex by learning, because these studies utilized steady state responses that reflect visual cortex activity across several seconds before US appearance $\mathrm{e}^{5,6,10}$. However, Keil et al. ${ }^{21}$ also reported gamma band increases between $60 \mathrm{~ms}$ and $90 \mathrm{~ms}$ after CS + onset during fear conditioning of visual gratings paralleling our results. In line with our study, Keil and collaborators $^{21}$ also observed the biggest learning induced gamma band modulations during the second acquisition block indicating increased neural gain for the fear relevant stimulus as a result of ongoing learning. However, these authors did not report inhibitory effects on gamma power for the CS-. This might be due to the utilization of unpleasant pictures as US. Here, we used a $90 \mathrm{~dB}$ SPL noise that may be more aversive and therefore probably induced more differential effects with respect to opposing neural gain modulations of the CS + and CS- in the visual cortex.

Conditioned inhibition of the gamma band response for the fear irrelevant CS- as observed here accords with animal studies in the auditory domain that showed similar opposing activity changes for the CS + and CS- in the amygdala, thalamus and auditory cortex ${ }^{22-24}$. At the cortical level these processes have been associated with frequency tuning shifts of neurons in the auditory system. Further, Headley and Weinberger ${ }^{17}$ have shown that neurons that code the tone frequency of the CS + exert gamma frequency boosts associated with increased phase-locking of single spike trains to gamma cycles. In contrast, neurons that code frequencies far from the $\mathrm{CS}+$ decreased in phase-locking of spike occurrence. In line with our observation of increased CS + gamma power and diminished CS- gamma band activity the opposing phase-locking patterns reported by Headley and Weinberger ${ }^{17}$ may represent a mechanism of a sharpened representation and down-stream processing of behaviorally relevant stimuli in comparison to irrelevant ones. Importantly, increased CS + gamma band oscillations after learning predicted the strength of associative memory and receptive field plasticity underscoring the behavioral relevance of these processes $^{16}$.

Critically, Galuske, Munk, and Singer ${ }^{7}$ observed a similar mechanism in the cat's visual cortex showing that cholinergic driven increased gamma band activity predicted changes of orientation preference in neural populations. The spatial extension of conditioned orientation domains was not only associated with increased gamma band responses to a conditioned orientation but also with a decrease of signal strength for the orthogonal orientation paralleling the opposing gamma power modulations in our study. It is likely that these processes are driven by disinhibition and inhibition of sensory cortex circuits modulated by cholinergic input from the basal forebrain during CS-US association learning ${ }^{8}$.

Interestingly, conditioned excitation and inhibition of gamma power changes in the visual cortex for the CS + and CS-, respectively, cannot only be explained by neurobiological informed models, but also formalized by classic learning theories ${ }^{25}$. The early Rescorla-Wagner model of how associative strength develops during the CS-US association learning and results in excitatory responses for the CS + also emphasizes the acquisition of conditioned inhibitory properties of the CS- ${ }^{26-28}$. Thereby, the final associative strength $\lambda$ between the CS + and US must be ideally reach 1 . This $\lambda$ is approached during trial- 
by-trial learning that follows a decreasing linear function of the difference between the accumulative associative strength $V$ and this predetermined $\lambda$ value: $\Delta V=\Phi(\lambda-V)$, whereby $\Phi$ stands for US intensity. In contrast, the final associative strength for the CS- must be ideally 0 . However, as both, the CS- and the $\mathrm{CS}+$, are always presented together with the background and experimental context, this context is also reinforced during CS + presentation and gains some associative strength with respect to the US. Now, when the CS- is presented together with the context, the final accumulative associative strength $V$ for the CS- must be negative in order to counteract the conditioned excitatory context. This is how the CSconverts itself into a conditioned inhibitor as the Rescorla-Wagner model assumes that association learning encompasses stimulus combinations ${ }^{28}$.

Taken together, both, classic learning theory and neurobiological models of cholinergic driven plasticity changes in early sensory cortex can explain our observation of opposing gamma band power modulations in early visual cortex during fear conditioning of visual stimuli. Thereby, increased macroscopic neuromagnetic gamma band responses for the fear relevant sinus grating of a certain orientation may reflect shifts of preferred orientation selectivity of visual cortex neurons towards the fear relevant orientation ${ }^{7}$. This increased recruitment of neurons preferably firing for the fear conditioned orientation probably is accompanied by increased gamma power as measured at a macroscopic level. This process would also lead to a reduction of gamma power for the orthogonal oriented sinus gratings that are fear irrelevant. Importantly, this results in a sharpened sensorial representation of the CS + as neural activity is not only increased for the fear relevant but also decreased for the fear irrelevant CSCritically, we show that conditioned excitatory and inhibitory neural gain control for fear relevant and irrelevant stimuli, respectively, occurs very fast after stimulus onset in early visual cortex.

\section{Methods}

\section{Subjects.}

A sample of 30 volunteers ( 22 females, 25 right-handed, mean age 23.9 years, range 20 years to 37 years) participated in the study. All participants had normal or corrected to normal vision and no auditory anomalies. The study had full ethical approval from the local ethics committee (Comisión deontológica/ Ethics Committee, Facultad de Psicología, Universidad Complutense de Madrid) according to the Declaration of Helsinki and all participants gave written informed consent. We confirm that all methods were performed in accordance with the relevant guidelines and regulations following the Spanish and European law (Ley Orgánica 15/ 1999 de Protección de datos de Carácter Personal LOPD y Real Decreto 994/ 1999).

\section{Stimuli}

The experimental design consisted of a delay fear conditioning paradigm following immediate extinction divided in three phases: habituation, conditioning and extinction. The conditioned stimuli (CS) stimuli were two orthogonally oriented $\left(45^{\circ}\right.$ and $\left.135^{\circ}\right)$ circular sinusoidal gratings of maximum contrast and 
consisted of 7 cycles across a visual angle of $1.44^{\circ}$. In order to optimally activate the cortical generators of the primary visual cortex we followed the procedure as suggested by DiRusso et al ${ }^{29}$. Thereby, stimuli were placed along a polar arc that was equidistant $\left(2.62^{\circ}\right)$ from a central fixation point and located at a polar angle of $45^{\circ}$ below the horizontal meridian in the left or right visual field. We have chosen to stimulate the upper bank of the left and right visual cortex as these areas are better covered by MEG sensors.

The assignment of the two gratings (oriented at $45^{\circ}$ or $135^{\circ}$ ) to the CS + or CS- condition was counterbalanced across subjects. A white acoustic noise ( $90 \mathrm{~dB}$ SPL) with instantaneous onset was binaurally presented through an air tube system (Neuroscan, El Paso, TX) as the unconditioned stimulus (US). A centered black dot, subtending a visual angle of $0.17^{\circ}$, was depicted during the whole experiment as the fixation point. During the experiment the dot color could sometimes change to grey $(40 \%$ of maximum white luminance). All visual stimuli were presented in a magnetically shielded room by a video projector (Panasonic PT-D7700E) via a mirror system.

Procedure

After subjects were familiarized with the MEG chamber, a written informed consent was given and signed. Then, subjects were seated into the shielded room placing their head under the MEG helmet. Subjects were instructed to focus on the black dot in the center of the screen and to press a button with their right or left index finger (counter balanced across subjects) as soon as the black dot changed its color to grey.

The experiment consisted of a discriminative delayed fear conditioning procedure with a habituation, acquisition, and extinction phase. Before habituation subjects were informed that no loud noise would be delivered. During habituation $40 \mathrm{CS}+$ and $40 \mathrm{CS}$ - stimuli were presented in the left and right visual field, respectively, resulting in 80 trials per condition (CS + and CS-). Each CS was presented for $0.8 \mathrm{~s}$ and the inter-trial interval varied randomly between $4 \mathrm{~s}$ and $5 \mathrm{~s}$. During 30 randomly chosen inter-trial intervals the black dot color changed to grey requiring the participant's responses.

Before the acquisition phase participants were informed that from now on a white acoustic noise burst would be presented from time to time. The acquisition block was identical to the habituation procedure, except that the US was always paired with CS+. Thereby the US (duration $0.400 \mathrm{~s}$ ) was presented after $0.4 \mathrm{~s}$ after $\mathrm{CS}+$ onset and co-terminated together with the CS+. The CS- was never paired with the US during acquisition. After the acquisition phase the extinction block started immediately without any instructions. The extinction block was identical to the habituation phase. After the experiment the participants were asked if they could identify the CS-US contingency.

\section{MEG data acquisition and pre-processing.}

MEG data was continuously recorded $(600 \mathrm{~Hz}$ sample rate, $0.1 \mathrm{~Hz}$ to $200 \mathrm{~Hz}$ online-filter) using a 306channel (102 magnetometers, 204 orthogonal gradiometers pairs) system (Elekta-Neuromag ${ }^{\circledR}$ VectorView, Helsinki, Finland, 2005). However, only gradiometer pairs were used for the final analysis as gradiometers are less noisy and we were not interested in deep sources. For artifact monitoring the 
electrooculogram (EOG) was recorded with electrodes attached above, below, left to and right to the outer canthus. The electrocardiogram (ECG) was recorded with electrodes placed at the left mid clavicle and lower right rib bone. Additionally, one electrode was attached to the left earlobe serving as the ground electrode. EOG and ECG were recorded simultaneously using standard Au electrodes (NSC Electromedicina) with the same sample frequency and on-line filters as the MEG data.

MEG data were spatially filtered using the temporal signal space separation (tSSS) algorithm implemented in Neuromag ${ }^{\circledR}$ MEG software ${ }^{30}$. Eye blinks and cardiac artefacts were removed from data by independent component analysis (ICA-JADE) implemented in Brainstorm software ${ }^{31}$ (https://neuroimage.usc.edu/brainstorm). Noisy trials due to movement artifacts and horizontal eye movements as monitored by the EOG were determined by visual inspection and excluded from analysis. As we performed time-frequency analysis, the MEG data was not further off-line filtered. Then, peristimulus epochs of $1.8 \mathrm{~s}$ ( $1 \mathrm{~s}$ baseline and $0.8 \mathrm{~s}$ post-stimulus) were extracted for each condition (CS + and CS-), experimental phase (habituation, acquisition, and extinction) and each subject. Left and right visual field conditions were collapsed. As learning effects were of principal interest in this study, experimental phases were further divided into first and second trial blocks (first 40 , and second 40 trials). Finally, the second habituation, first and second acquisition and first and second extinction blocks entered into the analysis. The first trial of the acquisition and extinction conditions were removed from analysis.

Spectral MEG analysis of evoked gamma band modulations.

First, artefact free epochs for each condition (CS + and CS-), experimental phase (habituation block II, acquisition blocks I and II, and extinction blocks I and II) and participants were averaged in order to obtain evoked MEG responses. Then, Morlet wavelets (7 cycles) were convolved with the averaged epochs at each gradiometer from $15 \mathrm{~Hz}$ to $80 \mathrm{~Hz}$ in steps of $1 \mathrm{~Hz}$ in order to obtain evoked gamma band responses as we were only interested in early evoked gamma band modulations. Finally, evoked spectral perturbations within a $0.3 \mathrm{~s}$ post-stimulus interval were assessed by calculating power changes in $\mathrm{dB}$ with respect to $0.3 \mathrm{~s}$ baseline. The time-frequency analysis was performed using the fieldtrip toolbox ${ }^{32}$ (https://www.fieldtriptoolbox.org). Induced gamma band responses were also evaluated using the same method but applied to single epochs before averaging (see supplemental Material for details).

Cortical source analysis

The source reconstruction of evoked MEG data epochs was based on a forward model based on an overlapping spheres head model ${ }^{33}$ using a canonical cortical mesh (3003 vertices) from the Montreal Neurological Institute ${ }^{34}$. Only gradiometer data was used for the cortical source reconstruction. The inverse solution was estimated with a linearly constrained minimum variance (LCMV) beamformer as implemented in the Brainstorm toolbox ${ }^{31}$. The data co-variance matrix for the common spatial filter across all conditions and experimental phases was calculated on the post-stimulus interval. Accordingly, a noise co-variance matrix was determined also across all conditions and experimental phases for the pre-stimulus interval. Then, the averaged MEG data epochs were convoluted with the post-stimulus 
spatial filter and z-normalized using the noise co-variance matrix in order to obtain a time domain Pseudo Neural Activity Index ${ }^{31}$ (PNAI) at three orthogonally oriented dipoles at each cortical vertex. Then, a Fast Fourier Transform (FFT) was applied to each PNAI time series of the three orthogonal dipoles at each vertex and the norm across the three orientations served as a power estimate at each cortical vertex. However, the FFT was restricted to the first $0.180 \mathrm{~s}$ after stimulus onset where significant gamma band modulations were observed at the sensor level (see Results). Then, an FFT in cortical source space was also calculated on a $0.180 \mathrm{~s}$ baseline interval $(-0.380 \mathrm{~s}$ to $-0.200 \mathrm{~s})$ in order to estimate mean power changes in $\mathrm{dB}$ between $18.9 \mathrm{~Hz}$ to $71 \mathrm{~Hz}$ (see Results) with respect to baseline. Statistical Analysis

The aim of this study was to find an increase of CS + and a decrease of CS- gamma power during learning of the CS-US association. Following the directed hypothesis of an increase of CS+/ CSdifferences from habituation over acquisition and a decrease during extinction a quadratic $\mathrm{F}$ contrast ${ }^{35}$ was fitted to power changes $(\mathrm{dB})$ for each time and frequency bin of the Morlet wavelet analysis at occipital sensors. Thereby, condition differences (CS + minus CS-) for the five experimental phases (habituation II, acquisition I, acquisition II, extinction I and extinction II) were weighted by the contrast coefficients $-2.5,1,3,1$, and -2.5 assuming biggest CS + and CS- power differences during the second acquisition block.

A cluster-based permutation statistic was utilized in order to control for multiple comparisons. However, as early evoked gamma band responses are observed at posterior sensors, the analysis was restricted to occipital and parietal sensors (see Fig. 1B grey shaded sensors). Sensor clusters were formed when at least one adjacent time-frequency bin and spatial sensor neighbor indicated a significant quadratic $\mathrm{F}$ contrast fit (cluster alpha threshold of $p=0.05$ ). Then, the $F$ values were summed across the cluster. Thereafter, 10.000 random permutations between experimental phases for each MEG sensor under the Null hypothesis of no changes of CS + and CS- power differences across the five experimental blocks were done. At each permutation step the same aforementioned cluster rule was applied and the maximum $\mathrm{F}$ cluster sum entered into a permutation distribution. Finally, empirical observed timefrequency sensor triplet $F$ clusters with a sum exceeding the 97.5 percentile of the permutation distribution were considered as significant ${ }^{36}$. The permutation statistics was performed by using the fieldtrip toolbox. Exactly, the same procedure was applied to the induced gamma band responses at sensor space (see supplemental Material).

In cortical source space the same permutation statistics was used. However, as the data dimension had been reduced based on the time-frequency results at the sensor space level (mean power changes across $18.9 \mathrm{~Hz}$ and $71 \mathrm{~Hz}$ derived from an FFT of the first $0.18 \mathrm{~s}$ post-stimulus interval), only spatial $\mathrm{F}$ clusters of neighboring vertices of the canonical brain surface were considered during the permutation process. However, here a cluster alpha threshold of $p=0.01$ was applied.

\section{Declarations}


Author contributions statement: ASM designed, conducted and analyzed the experiments and wrote the manuscript. JE conducted and analyzed the experiments. SM designed, conducted and analyzed the experiments. Further, SM wrote the manuscript and obtained funding for the project.

Acknowledgements: We thank the Ministerio de Ciencia e Innovación for funding the current study (PGC2018-097940-B-I00). Further, AMS was funded by a FPI grant associated with the aforementioned funding. JE was funded by a pre-doctoral stipend of the Universidad Complutense de Madrid and Santander bank.

Additional information: The authors declare no competing interests.

\section{Data availability:}

The data and analysis scripts can be obtained from the authors upon reasonable request.

\section{References}

1. Öhman, A., Flykt, A. \& Esteves, F. Emotion drives attention: detecting the snake in the grass. $J$ Exp Psychol Gen, 130, 466-478 https://doi.org/10.1037//0096-3445.130.3.466 (2001).

2. Ohman, A. \& Mineka, S. Fears, phobias, and preparedness: toward an evolved module of fear and fear learning. Psychol Rev, 108, 483-522 https://doi.org/10.1037/0033-295x.108.3.483 (2001).

3. Ohman, A. \& Soares, J. J. Emotional conditioning to masked stimuli: expectancies for aversive outcomes following nonrecognized fear-relevant stimuli. J Exp Psychol Gen, 127, 69-82 https://doi.org/10.1037//0096-3445.127.1.69 (1998).

4. Miskovic, V. \& Keil, A. Acquired fears reflected in cortical sensory processing: a review of electrophysiological studies of human classical conditioning., 49, 1230-1241 https://doi.org/10.1111/j.1469-8986.2012.01398.x (2012).

5. Moratti, S., Gimenez-Fernandez, T., Mendez-Bertolo, C. \& de Vicente-Perez, F. Conditioned inhibitory and excitatory gain modulations of visual cortex in fear conditioning: Effects of analysis strategies of magnetocortical responses., 54, 882-893 https://doi.org/10.1111/psyp.12841 (2017).

6. Moratti, S., Keil, A. \& Miller, G. A. Fear but not awareness predicts enhanced sensory processing in fear conditioning., 43, 216-226 https://doi.org/10.1111/j.1464-8986.2006.00386.x (2006).

7. Galuske, R. A. W., Munk, M. H. J. \& Singer, W. Relation between gamma oscillations and neuronal plasticity in the visual cortex. Proc Natl Acad Sci U S A, 116, 23317-23325 https://doi.org/10.1073/pnas.1901277116 (2019).

8. Letzkus, J. J. et al. A disinhibitory microcircuit for associative fear learning in the auditory cortex. Nature, 480, 331-335 https://doi.org/10.1038/nature10674 (2011).

9. McGann, J. P. Associative learning and sensory neuroplasticity: how does it happen and what is it good for? Learn Mem, 22, 567-576 https://doi.org/10.1101/Im.039636.115 (2015). 
10. McTeague, L. M., Gruss, L. F. \& Keil, A. Aversive learning shapes neuronal orientation tuning in human visual cortex. Nat Commun, 6, 7823 https://doi.org/10.1038/ncomms8823 (2015).

11. Keil, A. et al. Re-entrant projections modulate visual cortex in affective perception: evidence from Granger causality analysis. Hum Brain Mapp, 30, 532-540 https://doi.org/10.1002/hbm.20521 (2009).

12. Basar-Eroglu, C., Struber, D., Schurmann, M., Stadler, M. \& Basar, E. Gamma-band responses in the brain: a short review of psychophysiological correlates and functional significance. Int $J$ Psychophysiol, 24, 101-112 https://doi.org/10.1016/s0167-8760(96)00051-7 (1996).

13. Schadow, J. et al. Stimulus intensity affects early sensory processing: visual contrast modulates evoked gamma-band activity in human EEG. Int J Psychophysiol, 66, 28-36 https://doi.org/10.1016/j.jpsycho.2007.05.010 (2007).

14. David, O., Kilner, J. M. \& Friston, K. J. Mechanisms of evoked and induced responses in MEG/EEG., 31, 1580-1591 https://doi.org/10.1016/j.neuroimage.2006.02.034 (2006).

15. Tallon-Baudry, C. \& Bertrand, O. Oscillatory gamma activity in humans and its role in object representation. Trends in Cognitive Sciences, 3, 151-162 https://doi.org/10.1016/s13646613(99)01299-1 (1999).

16. Headley, D. B. \& Weinberger, N. M. Gamma-band activation predicts both associative memory and cortical plasticity. J Neurosci, 31, 12748-12758 https://doi.org/10.1523/JNEUROSCI.2528-11.2011 (2011).

17. Headley, D. B. \& Weinberger, N. M. Fear conditioning enhances gamma oscillations and their entrainment of neurons representing the conditioned stimulus. J Neurosci, 33, 5705-5717 https://doi.org/10.1523/JNEUROSCI.4915-12.2013 (2013).

18. Weinberger, N. M. New perspectives on the auditory cortex: learning and memory. Handb Clin Neurol, 129, 117-147 https://doi.org/10.1016/B978-0-444-62630-1.00007-X (2015).

19. Herculano-Houzel, S., Munk, M. H., Neuenschwander, S. \& Singer, W. Precisely synchronized oscillatory firing patterns require electroencephalographic activation. J Neurosci, 19, 3992-4010 https://doi.org/10.1523/JNEUROSCl.19-10-03992.1999 (1999).

20. Rodriguez, R., Kallenbach, U., Singer, W. \& Munk, M. H. Short- and long-term effects of cholinergic modulation on gamma oscillations and response synchronization in the visual cortex. $J$ Neurosci, 24, 10369-10378 https://doi.org/10.1523/JNEUROSCl.1839-04.2004 (2004).

21. Keil, A., Stolarova, M., Moratti, S. \& Ray, W. J. Adaptation in human visual cortex as a mechanism for rapid discrimination of aversive stimuli., 36, 472-479 https://doi.org/10.1016/j.neuroimage.2007.02.048 (2007).

22. Bakin, J. S. \& Weinberger, N. M. Classical conditioning induces CS-specific receptive field plasticity in the auditory cortex of the guinea pig. Brain Res, 536, 271-286 https://doi.org/10.1016/00068993(90)90035-a (1990).

23. Collins, D. R. \& Pare, D. Differential fear conditioning induces reciprocal changes in the sensory responses of lateral amygdala neurons to the CS(+) and CS(-). Learn Mem, 7, 97-103 
https://doi.org/10.1101/lm.7.2.97 (2000).

24. Edeline, J. M. \& Weinberger, N. M. Associative retuning in the thalamic source of input to the amygdala and auditory cortex: receptive field plasticity in the medial division of the medial geniculate body. Behav Neurosci, 106, 81-105 https://doi.org/10.1037//0735-7044.106.1.81 (1992).

25. Yuan, M., Gimenez-Fernandez, T., Mendez-Bertolo, C. \& Moratti, S. Ultrafast Cortical Gain Adaptation in the Human Brain by Trial-To-Trial Changes of Associative Strength in Fear Learning. J Neurosci, 38, 8262-8276 https://doi.org/10.1523/JNEUROSCI.0977-18.2018 (2018).

26. Rescorla, R. A. Pavlovian Conditioned Inhibition. Psychol. Bull, 72, 77-94 https://doi.org/10.1037/h0027760 (1969).

27. Rescorla, R. A. \& Pavlovian conditioning. It's not what you think it is. Am Psychol 43, 151-160, doi: 10.1037//0003-066x.43.3.151 (1988).

28. Wagner, A. R. \& Rescorla, R. A. Inhibition in Pavlovian conditioning: Application of a theory. In Inhibition and Learning (eds R. A. Boaks \& M.S. Halliday) (Academic Press, Inc., 1972).

29. Di Russo, F., Martinez, A., Sereno, M. I., Pitzalis, S. \& Hillyard, S. A. Cortical sources of the early components of the visual evoked potential. Hum Brain Mapp, 15, 95-111 https://doi.org/10.1002/hbm.10010 (2002).

30. Taulu, S. \& Hari, R. Removal of magnetoencephalographic artifacts with temporal signal-space separation: demonstration with single-trial auditory-evoked responses. Hum Brain Mapp, 30, 15241534 https://doi.org/10.1002/hbm.20627 (2009).

31. Tadel, F., Baillet, S., Mosher, J. C., Pantazis, D. \& Leahy, R. M. Brainstorm: a user-friendly application for MEG/EEG analysis. Comput Intell Neurosci 2011, 879716, doi:10.1155/2011/879716 (2011).

32. Oostenveld, R., Fries, P., Maris, E., Schoffelen, J. M. \& FieldTrip Open source software for advanced analysis of MEG, EEG, and invasive electrophysiological data. Comput Intell Neurosci 2011, 156869, doi:10.1155/2011/156869 (2011).

33. Huang, M. X., Mosher, J. C. \& Leahy, R. M. A sensor-weighted overlapping-sphere head model and exhaustive head model comparison for MEG. Phys Med Biol, 44, 423-440 https://doi.org/10.1088/0031-9155/44/2/010 (1999).

34. Collins, D. L. et al. Design and construction of a realistic digital brain phantom. IEEE Trans Med Imaging, 17, 463-468 https://doi.org/10.1109/42.712135 (1998).

35. Rosenthal, R. \& Rosnow, R. Contrast analysis: Focused comparisons in the analysis of variance (CUP, 1985).

36. Nichols, T. E. \& Holmes, A. P. Nonparametric permutation tests for functional neuroimaging: a primer with examples. Hum Brain Mapp, 15, 1-25 https://doi.org/10.1002/hbm.1058 (2002).

\section{Figures}


A
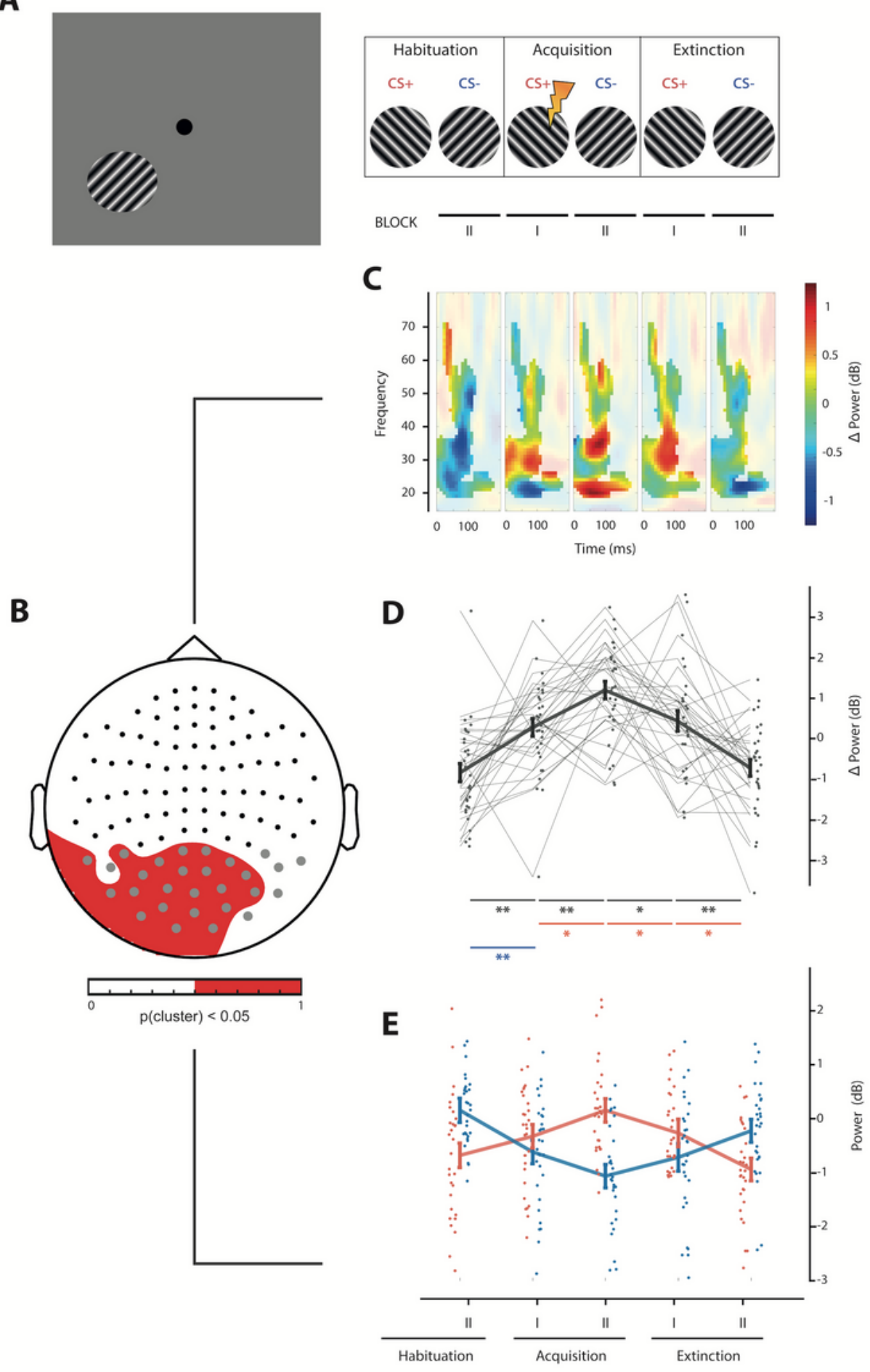

\section{Figure 1}

Sensor space analysis of evoked gamma band power. (A) The left panel represents the lower left visual field presentation of a sinusoidal grating. The right panel depicts the three phases of the experiment (habituation, acquisition, and extinction). Each phase was divided into two blocks. (B) The topography of the significant quadratic contrast cluster of the CS+/ CS- differences across experimental phases is shown. The colorbar indicates in red which sensors pertain to the cluster (pcluster < 0.05). (C) Mean 
spectral power differences (CS+ minus CS-) in the time-frequency domain across significant cluster sensors for each experimental phase are shown. The colorbar represents power changes in dB. (D) Mean power differences (CS+ minus CS-) across significant sensor, time, and frequency triplet clusters are shown (grey line). The error bars represent standard errors. Further, a paired observation plot for each subject (connected dots, $\mathrm{N}=30$ ) was overlayed to the contrast plot. (E) Mean power changes $(\mathrm{dB})$ to a pre-stimulus baseline across the same sensor, time, and frequency triplet clusters as in $D$ are shown for the CS+ (red) and CS- (blue) conditions separately and for each block. The dots represent individual participants $(\mathrm{N}=30)$. The error bars represent standard errors.

A
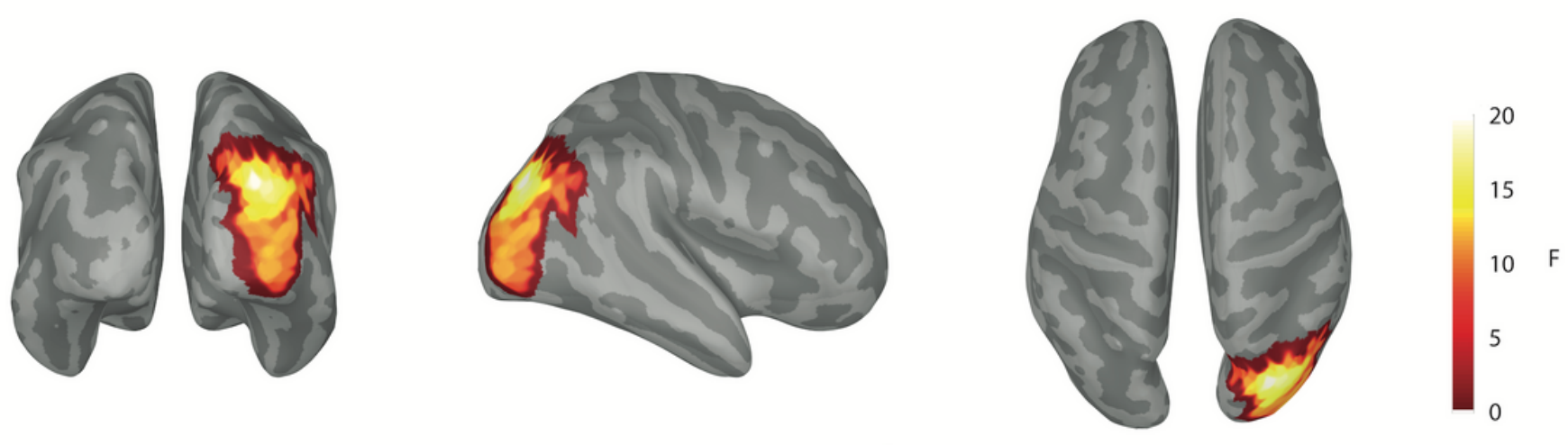

B

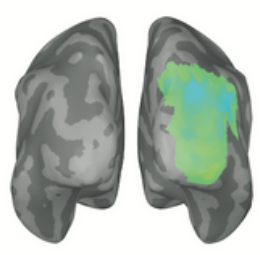

Habituation II

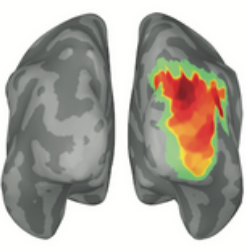

Acquisition I

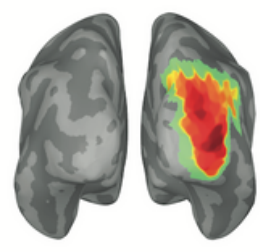

Acquisition II

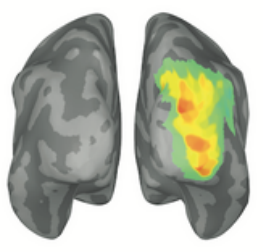

Extinction I

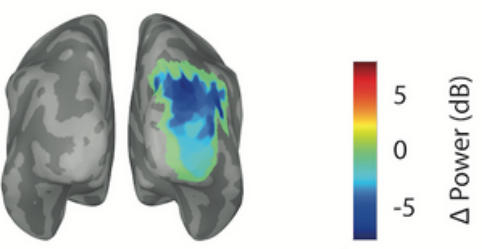

Extinction II

\section{Figure 2}

Source space analysis of evoked gamma power. (A) The significant cluster of the quadratic fit for the $\mathrm{CS}+/$ CS- differences across experimental blocks is shown (back, right lateral and top view). The colorbar indicates the $F$ value of the quadratic contrast for the CS+ and CS- power differences across experimental phases. (B): Gamma power response differences between the CS+ and the CS- for sources within the aforementioned significant cluster across experimental blocks are depicted. The colorbar represents gamma power $\mathrm{CS}+/ \mathrm{CS}$-differences in $\mathrm{dB}$.

\section{Supplementary Files}

This is a list of supplementary files associated with this preprint. Click to download.

- SupplementaryInformationSantosMayosubmission.pdf 